\title{
An equi-biased $k$-prototypes algorithm for clustering mixed-type data
}

\author{
RAVI SANKAR SANGAM* and HARI OM \\ Department of Computer Science and Engineering, Indian Institute of Technology (ISM), Dhanbad, \\ Jharkhand 826004, India \\ e-mail: srskar@gmail.com; hariom4india@gmail.com
}

MS received 3 June 2014; revised 1 February 2018; accepted 6 February 2018; published online 14 March 2018

\begin{abstract}
Clustering has been recognized as a very important approach for data analysis that partitions the data according to some (dis)similarity criterion. In recent years, the problem of clustering mixed-type data has attracted many researchers. The $k$-prototypes algorithm is well known for its scalability in this respect. In this paper, the limitations of dissimilarity coefficient used in the $k$-prototypes algorithm are discussed with some illustrative examples. We propose a new hybrid dissimilarity coefficient for $k$-prototypes algorithm, which can be applied to the data with numerical, categorical and mixed attributes. Besides retaining the scalability of the $k$ prototypes algorithm in our method, the dissimilarity functions for either-type attributes are defined on the same scale with respect to their dimensionality, which is very beneficial to improve the efficiency of clustering result. The efficacy of our method is shown by experiments on real and synthetic data sets.
\end{abstract}

Keywords. Data clustering; data mining; $k$-prototypes; similarity coefficient.

\section{Introduction}

In scientific areas such as data mining [1], pattern recognition [2], medical diagnosis [3], data streams [4], data compression [5] and information retrieval [6], the clustering analysis has been recognized as an important approach. Clustering is a process of dividing a data set into mutually exclusive groups based on some similarity criterion such that the members of each group are as 'close' as possible to one another and the different groups are as 'far as possible from one another [7]. Here the similarity between any two data points is usually measured by some distance function, wherein its higher value represents lower similarity and vice versa. Many traditional clustering algorithms are applicable to either numerical data or categorical data only, but not for both, since the objective functions of clustering algorithms are defined only for numerical or categorical attributes. Some of the examples include $k$ means $[8,9]$, fuzzy $c$ means [10], $k$ modes [11], fuzzy $k$ modes [12], ROCK [13] and COOLCAT [14]. The real world data sets usually contain both numerical and categorical data and hence these methods cannot be applied on such data sets. One simple approach to handle the mixed data is to convert one type of data to another type and then apply a singletype clustering algorithm on the transformed data [15]. The transformation of data may result in loss of useful information and does not reveal the original similarity structure of the data set [16].

*For correspondence
In $[17,18]$ the $k$-prototypes clustering algorithm is proposed, an extension to the $k$-mean algorithm, to deal with the mixed-type data by combining the $k$-modes [11] and $k$ means [9] algorithms. This algorithm is popular due to its scalability, simplicity and speed of convergence. The problem with this algorithm is that the distance metrics for categorical part and numerical part are not defined on the same scale. To maintain the equality proportions for eithertype attributes, the user needs to specify a weightage parameter, which is not trivial when no prior knowledge is available, and also the final clustering result is sensitive to this weightage parameter. Nevertheless, the simple matching distance metric defined for the categorical part is not a good measure for high-dimensional data, since the simple hamming distance between any pair of high-dimensional data points is almost the same, which often results in poor intra-cluster similarity. Based on the foregoing discussion, for the mixed data domain, the problem of finding resemblance between any two data points is still a challenging issue, since there is an awkward gap between numerical and categorical attributes. Despite the fact that the $k$-prototypes algorithm was proposed over 15 years ago and a number of clustering algorithms have appeared for mixed data since then, the $k$-prototypes is still being widely used. In this paper, we propose a new dissimilarity metric for the $k$ prototypes algorithm to find (dis)similarity between two data points described by the mixed-type attributes. Based on the Minkowski distance metric, a dissimilarity coefficient is defined for numerical attributes. The original simple matching distance metric for categorical part is replaced 
with the proposed weightage hamming dissimilarity metric. This distance metric considers both the distribution and relative frequency of each mode category. The proposed dissimilarity metric for mixed data has the following advantages.

- The $k$-prototypes algorithm with new dissimilarity metric exhibits high cluster accuracy while retaining its original scalability.

- The new dissimilarity metrics for categorical and numerical attributes are defined on the same scale (i.e., equally biased either-type attributes), which is very beneficial for clustering large data sets.

- The proposed dissimilarity coefficient preserves the characteristics of clustering, i.e., both high intra-cluster similarity and low inter-cluster similarity.

The rest of the paper is organized as follows. In section 2, the related works are reviewed, and in section 3, various notations and the $k$-prototypes algorithm are presented. Section 4 explains the proposed method and section 5 illustrates its efficiency. Finally, we draw the conclusion in section 6 .

\section{Related works}

Here, we focus on reviewing some of the clustering algorithms, which are applicable to mixed data. However, detailed surveys on traditional clustering algorithms can be found in [19-22]. Based on the Goodall similarity metric [23], the Similarity-Based Agglomerative Clustering (SBAC) algorithm is discussed in [16]. This method is very effective to deal with the mixed-type data, but the computational cost is quite high. In [24], an extended version of the Squeezer algorithm [25] is proposed to cluster the mixed data in which the similarity metric for categorical part is the same as that of the original Squeezer algorithm. In this work, based on the relative difference, a new distance metric is defined for numerical part, but its feasibility has not been demonstrated sufficiently. In [26], an automated technique is presented to handle mixed data in which all the numerical attribute values are transformed into categorical values. However, doing this does not reveal the original similarity structure of the data sets. In [27], an Evidence-Based Spectral Clustering algorithm is proposed for mixed data by integrating the evidence-based similarity measure into spectral clustering structure. The algorithm [28] assumes a classical finite mixture distribution model on mixed data and utilizes a Bayesian model to derive the most probable class distribution for the data given with prior information. In [29], a distance metric for mixed-type attributes is discussed, which is derived from the probabilistic model in which the distance between two clusters is equivalent to the decrease in the log-likelihood function as for merging. However, in this metric the similarity between clusters with respect to numerical and categorical attributes is quantified with the same distance metric, which does not reveal the original similarity structure of the data points. Based on the information theorem (entropy), in [30], a resemblance coefficient to find the similarity between a data point and cluster centre is discussed. The similarity value between any two data points depends on uncertainty of the attribute values. Nonetheless, the computational cost of this method is quite high. In [15], a Clustering Algorithm based on the Variance and Entropy (CAVE) is discussed for clustering mixed data with application to catalog marketing data. However, in this algorithm a distance hierarchy for every categorical attribute needs to be built that can be done only by a domain expert. In [31], an unified similarity coefficient and an iterative clustering algorithm to deal with the mixed-type data are proposed. The similarity coefficient is constructed based on the importance of the categorical attribute values, but its scalability has not been demonstrated sufficiently. Besides, the unified similarity coefficient is not defined with respect to the dimensionality of either-type attributes. Among the afore-mentioned algorithms, the $k$-prototypes algorithm [18] is very cost-effective, in which a simple matching distance metric is adopted for categorical attributes and the numerical attributes are quantified with the squared Euclidean distance. Since two different distance metrics are adopted for numerical and categorical data, a user-specified weightage parameter is used to balance the magnitudes of distance metrics. Nevertheless, the final clustering result is very sensitive to the user-specified parameter. In [32], a modified version of $k$ prototypes algorithm is introduced to improve the clustering accuracy of the $k$-prototypes algorithm in which the categorical parts of clusters are represented with the distributed centroids rather than the simple modes. This method also requires a user-specified weightage parameter. In this category also, an efficient method is required to cluster the mixed data effectively. As a result, we propose a new distance function in $k$-prototypes algorithm that improves the accuracy of cluster results while retaining its scalability.

\section{Notations and $k$-prototypes algorithm}

We first introduce some of the notations used throughout the paper and then discuss the original $k$-prototypes algorithm.

\subsection{Notations}

For ease of representation and better understandability of algorithm, we consider a data set $D=\left\{x_{1}, x_{1}, \ldots, x_{n}\right\}$, where $x_{j}, 1 \leq j \leq n$, is $j$ th data point with an array of $m$ attributes, i.e., $\quad x_{j}=\left\{A_{1, j}^{c}, A_{2, j}^{c}, \ldots, A_{p, j}^{c}, A_{p+1, j}^{n}, \ldots, A_{m, j}^{n}\right\}$; here $A_{s, j}^{c}, 1 \leq s \leq p$, is sth categorical attribute of $j$ th data 
point that possesses one value from the domain of categorical values; the domain of categorical values of an sth attribute is represented by $\operatorname{DOM}\left(A_{s}^{c}\right)$ and $A_{s, j}^{c}$, $p+1 \leq s \leq m$, is sth numerical attribute of $j$ th data point. We assume that every data point in data set is described by exactly $m$ attributes. The objective of the clustering algorithm can be stated as 'divide the data set $D$ into $k$ distinct groups (clusters) such that the members of each group are as close as possible to one another and the different groups are as far as possible from one another'.

\section{2 k-prototypes algorithm}

The $k$-prototypes algorithm, through the definition of a combined dissimilarity measure, integrates the $k$-means and $k$-modes algorithms to allow for clustering data points described by mixed numeric and categorical attributes [18]. Let $x_{1}$ and $x_{2}$ be two data points in their mixed attribute space. The dissimilarity (distance) between these two data points is defined as follows:

$$
\operatorname{dist}\left(x_{1}, x_{2}\right)=\sum_{s=p+1}^{m}\left(A_{s, 1}^{n}-A_{s, 2}^{n}\right)^{2}+\gamma \sum_{s=1}^{p} d_{c}\left(A_{s, 1}^{c}, A_{s, 2}^{c}\right)
$$

where the first part of the equation is squared Euclidean distance on numerical attributes and the second part is simple matching distance on categorical attributes. The variable $\gamma$ is a user-specified parameter to balance the proportions of two distance functions. The simple matching distance between two categorical attributes is defined as follows:

$$
d_{c}\left(A_{s, 1}^{c}, A_{s, 2}^{c}\right)= \begin{cases}0 & \text { if } A_{s, 1}^{c}=A_{s, 2}^{c} \\ 1 & \text { if } A_{s, 1}^{c} \neq A_{s, 2}^{c} .\end{cases}
$$

The objective of $k$-prototypes algorithm is to divide the data set into $k$ clusters by minimizing the cost function as given in the following equation:

$$
C(W, Q)=\sum_{i=1}^{k} \sum_{j=1}^{n} w_{j i} \operatorname{dist}\left(x_{j}, q_{i}\right)
$$

where $x_{j}$ is $j$ th data point from $D$ and $q_{i}$ is prototype of $i$ th cluster; $w_{j i} \in(0,1)$ is an element of partition matrix $W_{(n \times k)}$. Based on foregoing discussion, the procedure of $k$-prototypes is described as follows:

1. Choose an initial prototype set $Q^{(1)} \in D$. Find $W^{(1)}$ such that $C\left(W, Q^{(1)}\right)$ is minimized. Set $t=1$.

2. Find $Q^{(t+1)}$ such that $C\left(W^{(t)}, Q^{(t+1)}\right)$ is minimized. If $C\left(W^{(t)}, Q^{(t+1)}\right)=C\left(W^{(t)}, Q^{(t)}\right)$, then stop; otherwise go to step 3.
3. Find $W^{(t+1)}$ such that $C\left(W^{(t+1)}, Q^{(t+1)}\right)$ is minimized. If $C\left(W^{(t+1)}, Q^{(t+1)}\right)=C\left(W^{(t)}, Q^{(t+1)}\right)$, then stop; otherwise, set $t=t+1$ and go to step 2 .

\subsection{Influence of user-specified threshold $(\gamma)$}

Here, we explore the drawback of the user-specified threshold with the help of artificial data set shown in table 1 . The artificial data set $D_{1}$ contains a total of 22 data points and each data point is exactly described by two numerical attributes and one categorical attribute. Here the categorical attribute $\left(A_{3}\right)$ possesses two categorical values, i.e., $\operatorname{DOM}\left(A_{3}\right)=\{C, T\}$. The numerical attributes $A_{1}$ and $A_{2}$ are in the range of $0-2$ and $10-30$, respectively.

For the threshold value $\gamma$ as zero, clustering on data set $D_{1}$, i.e., clustering result will depend on only the locations of numerical attributes as shown in figure 1. It may be noted that in figure 1 the data points that owe categorical value ' $\mathrm{C}$ ' for the attribute $A_{3}$ are represented with solid circle, the data points that owe categorical value ' $\mathrm{T}$ ' for the attribute $A_{3}$ are represented with solid triangle and the result will be the two clusters, which are separated by a dashed vertical line. If $\gamma>0$, then the data point $x_{7}$ may move to cluster 2 since most of the data points in that cluster have the same categorical value for attribute $A_{3}$. Similarly, the data point $x_{19}$ may move to cluster 1 due to the aforesaid reason. The data points $x_{7}$ and $x_{19}$ become uncertain depending on whether the threshold value $\gamma$ is biased towards the

Table 1. Artificial data set $D_{1}$.

\begin{tabular}{lccc}
\hline Data point & $A_{1}$ & $A_{2}$ & $A_{3}$ \\
\hline$x_{1}$ & 1.58 & 23.5 & $\mathrm{C}$ \\
$x_{2}$ & 0.62 & 21 & $\mathrm{~T}$ \\
$x_{3}$ & 0.15 & 19.8 & $\mathrm{C}$ \\
$x_{4}$ & 1.73 & 21.2 & $\mathrm{~T}$ \\
$x_{5}$ & 0.42 & 22.3 & $\mathrm{~T}$ \\
$x_{6}$ & 1.24 & 20 & $\mathrm{C}$ \\
$x_{7}$ & 0.9 & 15.8 & $\mathrm{C}$ \\
$x_{8}$ & 1.45 & 24.3 & $\mathrm{C}$ \\
$x_{9}$ & 0.25 & 18 & $\mathrm{~T}$ \\
$x_{10}$ & 1.29 & 17.5 & $\mathrm{C}$ \\
$x_{11}$ & 0.4 & 16.4 & $\mathrm{~T}$ \\
$x_{12}$ & 1.52 & 21.1 & $\mathrm{C}$ \\
$x_{13}$ & 0.46 & 19.4 & $\mathrm{~T}$ \\
$x_{14}$ & 1.34 & 22.5 & $\mathrm{C}$ \\
$x_{15}$ & 0.57 & 17 & $\mathrm{~T}$ \\
$x_{16}$ & 1.61 & 18.2 & $\mathrm{C}$ \\
$x_{17}$ & 1.32 & 25 & $\mathrm{C}$ \\
$x_{18}$ & 0.28 & 23.2 & $\mathrm{~T}$ \\
$x_{19}$ & 1.12 & 23 & $\mathrm{~T}$ \\
$x_{20}$ & 0.75 & 18.3 & $\mathrm{~T}$ \\
$x_{21}$ & 1.46 & 24.3 & $\mathrm{C}$ \\
$x_{22}$ & 0.48 & & $\mathrm{~T}$ \\
\hline & &
\end{tabular}




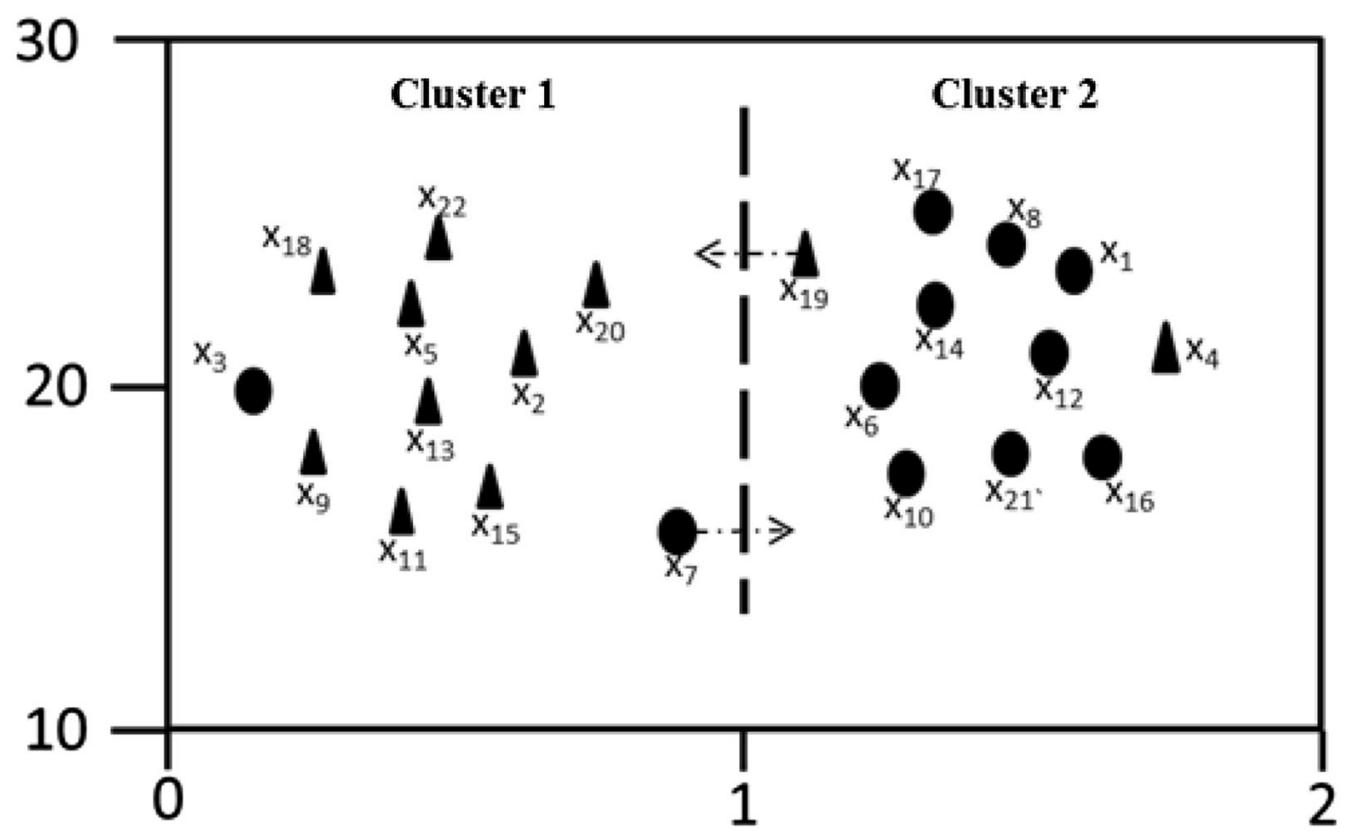

Figure 1. Clustering result of artificial data set $D_{1}$ with respect to numerical attributes.

numerical part or categorical part. The data points $x_{3}$ and $x_{4}$ may still remain in the same cluster because they are too far to the opposite side clusters, even though they have categorical values that are same as those of the majority data points in the opposite side clusters. Based on this discussion, it is very important to define the distance functions for either-type attributes on the same scale with respect to their dimensionality.

\section{Proposed scheme}

We first discuss the dissimilarity coefficient for data points described by categorical attributes followed by the dissimilarity method for data points based on the Minkowski distance metric described by numerical attributes. The discussion is further augmented by a hybrid dissimilarity coefficient for the mixed-type data points. Section 4.1 describes dissimilarity coefficient for categorical attributes.

\subsection{New dissimilarity coefficient for categorical attribute domain}

In this section, we first discuss the drawback of simple matching distance metric adopted for categorical attributes in original $k$-prototypes algorithm with some illustrative examples. This distance metric does not consider the relative frequencies of attribute values in each cluster mode. This results in poor intra-cluster similarity by allocating less similar data points to a cluster.

Let us consider the artificial data set $D_{2}$ as shown in figure 2 to demonstrate the aforesaid problem. The data set is described by three categorical attributes $\left(A_{1}, A_{2}\right.$, and $\left.\mathrm{A}_{3}\right)$ and grouped into two clusters with two modes $\left(q_{1}, q_{2}\right)$. Suppose we have to allocate data point $x_{1}[m, s, t]$ to any one cluster according to categorical part of distance metric (Eq. (1)) as given below:

$$
\operatorname{dist}_{c}\left(x_{1}, q_{1}\right)=0 \text {, and } \operatorname{dist}_{c}\left(x_{1}, q_{2}\right)=0 .
$$

According to the above calculated distance values, we can allocate $x_{1}$ to either cluster 1 or cluster 2 since the distance between data point and two modes is same. As far as intra-cluster similarity is concerned, it is more appropriate to assign $x_{1}$ to cluster1. To overcome this drawback, in [33, 34], introduced a new dissimilarity function in $k$ modes algorithm based on the relative frequency of categorical attribute values. The dissimilarity measure (Eq. (1)) with respect to the categorical domain is redefined as follows:

$$
\operatorname{dist}_{r}\left(x_{1}, q_{i}\right)=\sum_{s=1}^{p} d_{r}\left(A_{s, 1}^{c}, A_{s, q_{i}}^{c}\right)
$$

where $A_{s, q_{i}}^{c}$ is $s$ th mode category of $i$ th prototype and

$$
d_{r}\left(A_{s, 1}^{c}, A_{s, q_{i}}^{c}\right)=\left\{\begin{array}{cc}
1-\frac{\lambda_{i}^{s, r}}{\left|c_{i}\right|} & \text { if } \quad A_{s, 1}^{c}=A_{s, q_{i}}^{c}, \\
1 & \text { otherwise. }
\end{array}\right.
$$

Here $\left|c_{i}\right|$ is the number of data points in $i$ th cluster that is given by $\left|c_{i}\right|=\left|\left\{i \mid w_{j i}=1\right\}\right|$, and $\lambda_{i}^{s, r}$ is the number of data points that contain category $a_{c}^{s, r}$ in $s$ th attribute of $i$ th cluster and it is given by the relation

$$
\lambda_{i}^{s, r}=\left|\left\{w_{i j} \mid A_{s, 1}^{c}=A_{s, q_{i}}^{c}=a_{c}^{s, r}, w_{i j}=1\right\}\right| .
$$




\begin{tabular}{|c|c|c|}
\hline \multicolumn{3}{|c|}{ cluster1 } \\
\hline$A_{1}$ & $A_{2}$ & $A_{3}$ \\
\hline$m$ & $s$ & $t$ \\
\hline$q$ & $s$ & $t$ \\
\hline$m$ & $s$ & $t$ \\
\hline \multicolumn{3}{|c|}{$q_{1}[m, s, t]$} \\
\hline
\end{tabular}

\begin{tabular}{|c|c|c|}
\hline \multicolumn{3}{|c|}{ cluster2 } \\
\hline$A_{1}$ & $A_{2}$ & $A_{3}$ \\
\hline$m$ & $s$ & $t$ \\
\hline$q$ & $u$ & $r$ \\
\hline$p$ & $r$ & $y$ \\
\hline \multicolumn{3}{|c|}{$q_{2}[m, s, t]$} \\
\hline
\end{tabular}

Figure 2. Artificial data set $D_{2}$.

According to the dissimilarity function (Eq. (4)), the dominant level of mode category is considered in calculation of the dissimilarity measure. When the dominant of any category mode is $100 \%$, then we have $\left|c_{i}\right|=\lambda_{i}^{s, r}$. Ng et al [35] have proved that the cost function (Eq. (3)) with the dissimilarity measure (Eq. (4)) is minimized with respect to the categorical domain and guarantees for convergence of the clustering process. Consider again the artificial data set $D_{2}$ in figure 2 . The dissimilarity between data point $x_{1}$ and modes according to the dissimilarity function (Eq. (4)) is given as follows:

$$
\begin{aligned}
& \operatorname{dist}_{r}\left(x_{1}, q_{1}\right)=1-\frac{2}{3}+1-\frac{3}{3}+1-\frac{2}{3}=\frac{1}{3}=0.33, \\
& \operatorname{dist}_{r}\left(x_{1}, q_{2}\right)=1-\frac{2}{3}+1-\frac{2}{3}+1-\frac{3}{3}=\frac{2}{3}=0.66 .
\end{aligned}
$$

We observe that the data point $x_{1}$ is more similar to cluster 1 and hence it is assigned to cluster 1 . The dissimilarity function (Eq. (4)) considers the relative frequency of each mode category, which can be deemed as intra-cluster similarity. It however does not consider the distribution of each category of mode between the clusters; this often minimizes the intercluster distance. We explore this drawback with a following illustrative example. Consider the artificial data set $D_{3}$ shown in figure 3. The data set is described by three categorical attributes $\left(A_{1}, A_{2}\right.$ and $\left.\mathrm{A}_{3}\right)$ and grouped into three clusters with three modes $\left(q_{1}, q_{2}, q_{3}\right)$. Suppose we have to allocate data point $x_{2}[m, s, t]$ to any single cluster. According to the dissimilarity metric (Eq. (4)), we have

$$
\begin{aligned}
& \operatorname{dist}_{r}\left(x_{2}, q_{1}\right)=1-\frac{2}{3}+1-\frac{3}{3}+1-\frac{2}{3}=\frac{2}{3}=0.66, \\
& \operatorname{dist}_{r}\left(x_{2}, q_{2}\right)=1-\frac{2}{3}+1-\frac{2}{3}+1-\frac{3}{3}=\frac{2}{3}=0.66, \\
& \operatorname{dist}_{r}\left(x_{2}, q_{3}\right)=1+1-\frac{3}{3}+1=2 .
\end{aligned}
$$

\begin{tabular}{|c|c|c|}
\hline \multicolumn{3}{|c|}{ cluster1 } \\
\hline$A_{1}$ & $A_{2}$ & $A_{3}$ \\
\hline$m$ & $s$ & $t$ \\
\hline$q$ & $s$ & $t$ \\
\hline$m$ & $s$ & $r$ \\
\hline \multicolumn{2}{|c|}{$q_{1}[m, s, t]$} \\
\hline
\end{tabular}

\begin{tabular}{|c|c|c|}
\hline \multicolumn{3}{|c|}{ cluster2 } \\
\hline$A_{1}$ & $A_{2}$ & $A_{3}$ \\
\hline$m$ & $s$ & $t$ \\
\hline$q$ & $u$ & $t$ \\
\hline$m$ & $s$ & $t$ \\
\hline \multicolumn{3}{|c|}{$q_{2}[m, s, t]$} \\
\hline
\end{tabular}

\begin{tabular}{|c|c|c|}
\hline \multicolumn{3}{|c|}{ cluster3 } \\
\hline$A_{1}$ & $A_{2}$ & $A_{3}$ \\
\hline$p$ & $s$ & $z$ \\
\hline$q$ & $s$ & $z$ \\
\hline$q$ & $s$ & $r$ \\
\hline \multicolumn{3}{|c|}{$q_{3}[q, s, z]$} \\
\hline
\end{tabular}

Figure 3. Artificial data set $D_{3}$.
We observe that $x_{2}$ can be assigned to either cluster1 or cluster2. Thus, we cannot determine the assignment of $x_{2}$ properly. However as far as the cluster characteristics, i.e., both high intra-cluster similarity and low inter-cluster similarity, are concerned, it is most appropriate to assign $x_{2}$ to cluster 2 because it maximizes the distance between cluster1 and cluster2. As a result, we have defined a weightage hamming distance, i.e., we have assigned a weightage value to each mode category based on its relative frequency and distribution of mode category between the clusters. The dissimilarity coefficient is defined as follows:

$$
\operatorname{dist}_{h}\left(x_{1}, q_{i}\right)=\sum_{s=1}^{p} d_{h}\left(A_{s, 1}^{c}, A_{s, q_{i}}^{c}\right)
$$

where

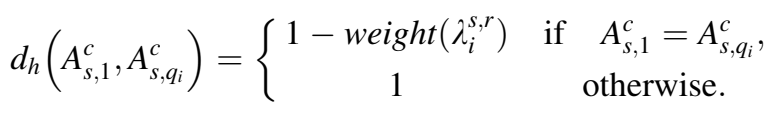

The weight is given by the relation

$$
\operatorname{weight}\left(\lambda_{i}^{s, r}\right)=\frac{\lambda_{i}^{s, r}}{\left|c_{i}\right|} \frac{\lambda_{i}^{s, r}}{\eta} .
$$

Here $\left|c_{i}\right|$ and $\lambda_{i}^{s, r}$ have the same meaning as in the dissimilarity function (Eq. (5)) and $\eta$ is the total number of data points that contain category $a_{c}^{s, r}$ in sth attribute in all clusters and it is given by the relation

$\eta=\sum_{i=1}^{k} \lambda_{i}^{s, r} \quad$ where $\lambda_{i}^{s, r}=\left|w_{i j}\right| A_{s, 1}^{c}=A_{s, q_{i}}^{c}=a_{c}^{s, r}, w_{i j}=1 \mid$.

It may be noted that the dissimilarity function (Eq. (6)) is defined in the interval $[0, p]$ where ' 0 ' indicates minimum distance (maximum similarity) and ' $p$ ' indicates maximum distance (minimum similarity). Consider again the artificial data set $D_{3}$ shown in figure 3. The dissimilarity between data point $x_{2}$ and three modes according to the dissimilarity function (Eq. (6)) is given as follows:

$$
\begin{aligned}
\operatorname{dist}_{h}\left(x_{2}, q_{1}\right)= & 1-\left(\frac{2}{3} \times \frac{2}{4}\right)+1-\left(\frac{3}{3} \times \frac{3}{8}\right) \\
& +1-\left(\frac{2}{3} \times \frac{2}{5}\right)=2.025 \\
\operatorname{dist}_{h}\left(x_{2}, q_{2}\right)= & 1-\left(\frac{2}{3} \times \frac{2}{4}\right)+1-\left(\frac{2}{3} \times \frac{2}{8}\right) \\
& +1-\left(\frac{3}{3} \times \frac{3}{5}\right)=1.9 \\
\operatorname{dist}_{h}\left(x_{2}, q_{3}\right)= & 1+1-\left(\frac{3}{3} \times \frac{3}{8}\right)+1=2.625 .
\end{aligned}
$$


The data point $x_{2}$ is more similar to cluster 2 and more dissimilar to cluster3 and cluster1; hence it is assigned to cluster2. We can conclude that the dissimilarity metric (Eq. (6)) considers both inter-cluster and intra-cluster similarity.

Theorem 1 Let $x_{1}, x_{2}$ and $x_{3}$ be three data points that are described by ' $p$ ' number of categorical attributes. Then the dissimilarity function given in Eq. (6) satisfies the following mathematical requirements of a dissimilarity (distance) function.

i. Nonnegative: $\operatorname{dist}_{h}\left(x_{1}, x_{2}\right) \geq 0$, and dist $t_{h}\left(x_{1}, x_{2}\right)=0$ if and only if $x_{1}=x_{2}$.

ii. Symmetric: $\operatorname{dist}_{h}\left(x_{1}, x_{2}\right)=\operatorname{dist}_{h}\left(x_{2}, x_{1}\right)$.

iii. Triangle inequality: $\operatorname{dist}_{h}\left(x_{1}, x_{2}\right) \leq \operatorname{dist}_{h}\left(x_{1}, x_{3}\right)+\operatorname{dist}_{h}\left(x_{3}, x_{2}\right)$.

Proof The proof of properties (i) and (ii) of the dissimilarity function $\operatorname{dist}_{h}\left(x_{1}, x_{2}\right)$ between two data points is trivial, which is an immediate consequence from its definition. To prove property (iii), we use the fact that when the weightage of sth categorical attribute value of any one data point is high, then it is obvious that for sth categorical attribute value of other two data points, the weightage will low, according to Eq. (8). Therefore, it always holds the triangle inequality for $s$ th categorical attribute, i.e., $d_{h}\left(A_{s, 1}^{c}, A_{s, 2}^{c}\right) \leq d_{h}\left(A_{s, 1}^{c}, A_{s, 3}^{c}\right)+d_{h}\left(A_{s, 3}^{c}, A_{s, 2}^{c}\right)$. Since it satisfies the triangle inequality for every sth attribute, it satisfies the same for the entire data point with dimensionality ' $\mathrm{p}$ '.

\subsection{New dissimilarity coefficient for numerical attribute domain}

The dissimilarity between two data points in their numerical attributes space can be quantified with any existing coefficients such as cosine similarity coefficient and Canberra similarity coefficient. However, these coefficients have certain drawbacks which are explained later in this section with an illustrative example. As a result, based on the Minkowski distance metric we define new dissimilarity measure for numerical attributes. When two data points $x_{1}$ and $x_{2}$ are viewed as two points in their numerical attribute space. The Minkowski distance between these two data points can be expressed as follows:

$$
D_{M}\left(x_{1}, x_{2}\right)=\left(\sum_{s=p+1}^{m}\left|A_{s, 1}^{n}-A_{s, 2}^{n}\right|^{r}\right)^{\frac{1}{r}} .
$$

Here ' $r$ ' can be any positive integer. It represents the Manhattan distance when $r=1$ and Euclidean distance when $r=2$ [36]. Now, the new dissimilarity coefficient is defined as follows:

$$
\operatorname{dist}_{n}\left(x_{1}, x_{2}\right)=\sum_{s=p+1}^{m} \frac{\left|A_{s, 1}^{n}-A_{s, 2}^{n}\right|}{\left|\operatorname{Gmax}\left(A_{s}^{n}\right)-\operatorname{Gmin}\left(A_{s}^{n}\right)\right|} .
$$

Here $\operatorname{Gmax}\left(A_{s}^{n}\right)$ and $\operatorname{Gmin}\left(A_{s}^{n}\right)$ are maximum and minimum values of sth numerical attribute, respectively. It may be noted that the dissimilarity between two data points is investigated individually and it is defined in the interval $[0, m-p]$. The dissimilarity value ' 0 ' indicates maximum similarity and the dissimilarity value ' $m-p$ ' indicates minimum similarity.

Theorem 2 Let $x_{1}, x_{2}$ and $x_{3}$ be three data points that are described with the dimensionality ' $p$ ' in their numerical attribute space. Then the dissimilarity function given in Eq. (11) satisfies the following dissimilarity (distance) metric properties.

i. $\quad$ Nonnegative: $\operatorname{dist}_{n}\left(x_{1}, x_{2}\right) \geq 0$, and dist $t_{n}\left(x_{1}, x_{2}\right)=0$ if and only if $x_{1}=x_{2}$.

ii. Symmetric: $\operatorname{dist}_{n}\left(x_{1}, x_{2}\right)=\operatorname{dist}_{n}\left(x_{2}, x_{1}\right)$.

iii. Triangle inequality:

$$
\operatorname{dist}_{n}\left(x_{1}, x_{2}\right) \leq \operatorname{dist}_{n}\left(x_{1}, x_{3}\right)+\operatorname{dist}_{n}\left(x_{3}, x_{2}\right) \text {. }
$$

Proof The proofs of properties (i) and (ii) are immediate consequence of the definition of dissimilarity function $\operatorname{dist}_{n}\left(x_{1}, x_{2}\right)$ between two data points. Property (iii) can be proved as follows.

The dissimilarity between $x_{1}$ and $x_{2}$ is given by the relation

$$
\operatorname{dist}_{n}\left(x_{1}, x_{2}\right)=\sum_{s=1}^{p} \frac{\left|A_{s, 1}^{n}-A_{s, 2}^{n}\right|}{\left|\operatorname{Gmax}\left(A_{s}^{n}\right)-G \min \left(A_{s}^{n}\right)\right|} .
$$

It can be written as follows:

$$
\begin{aligned}
& \sum_{s=1}^{p} \frac{\left|\left(A_{s, 1}^{n}-A_{s, 2}^{n}\right)+\left(A_{s, 3}^{n}-A_{s, 3}^{n}\right)\right|}{\left|\operatorname{Gmax}\left(A_{s}^{n}\right)-G \min \left(A_{s}^{n}\right)\right|} \\
\leq & \sum_{s=1}^{p} \frac{\left|A_{s, 1}^{n}-A_{s, 3}^{n}\right|+\left|A_{s, 3}^{n}-A_{s, 2}^{n}\right|}{\left|\operatorname{Gmax}\left(A_{s}^{n}\right)-G \min \left(A_{s}^{n}\right)\right|} \\
\leq & \sum_{s=1}^{p} \frac{\left|A_{s, 1}^{n}-A_{s, 3}^{n}\right|}{\left|\operatorname{Gmax}\left(A_{s}^{n}\right)-G \min \left(A_{s}^{n}\right)\right|} \\
& +\sum_{s=1}^{p} \frac{\left|A_{s, 3}^{n}-A_{s, 2}^{n}\right|}{\left|\operatorname{Gmax}\left(A_{s}^{n}\right)-G \min \left(A_{s}^{n}\right)\right|} \\
\leq & \operatorname{dist}_{n}\left(x_{1}, x_{3}\right)+\operatorname{dist}_{n}\left(x_{3}, x_{2}\right) .
\end{aligned}
$$

The proposed dissimilarity coefficient (Eq. (11)) has the following advantages over the cosine similarity coefficient and the Canberra similarity coefficient.

- Unlike the cosine similarity coefficient, it is insensitive to the proportional transitivity. 
- The Canberra similarity coefficient reveals the original similarity when the data points are described only by either negative or positive values. Unlike the Canberra similarity coefficient, the proposed coefficient works even when the data points are described by both positive and negative values.

Here, we first present these two similarity coefficients and then explain the advantages of our proposed coefficient over these two existing coefficients with an illustrative example. The cosine similarity coefficient is defined as follows:

$$
C_{s}\left(x_{1}, x_{2}\right)=\frac{\sum_{s=p+1}^{m} A_{s, 1}^{n} A_{s, 2}^{n}}{\sqrt{\left(\sum_{s=p+1}^{m}\left(A_{s, 1}^{n}\right)^{2}\right)} \sqrt{\left(\sum_{s=p+1}^{m}\left(A_{s, 2}^{n}\right)^{2}\right)}} .
$$

It is defined on the scale $[-1,1]$; the similarity value ' -1 ' indicates minimum similarity and the similarity value ' 1 ', indicates maximum similarity. The Canberra similarity coefficient is defined as follows:

$$
C_{b}\left(x_{1}, x_{2}\right)=\frac{1}{m-p} \sum_{s=p+1}^{m} \frac{\left|A_{s, 1}^{n}-A_{s, 2}^{n}\right|}{\left|A_{s, 1}^{n}\right|+\left|A_{s, 2}^{n}\right|} .
$$

It is defined on the scale $[0,1]$, where the dissimilarity value ' 1 ' indicates minimum similarity and the dissimilarity value ' 0 ' indicates maximum similarity.

Example 1 Consider the data points $X=\{10,23,17\}$, $Y=30 X$, i.e., $\{300,690,510\}$ and $Z=\{-10,27,-30\}$. The similarities between these data points are quantified with these three (dis)similarity coefficients, which are summarized in table 2. It is to be mentioned that all the calculated values are transformed into similarity values in the range $[0,1]$, where former value represents minimum similarity and latter value represents maximum similarity. From the summarized values, we see that the cosine similarity coefficient has ignored the size displacement between the data points $X, Y$ and has returned the maximum
Table 2. Summary of similarities among data points.

\begin{tabular}{llll}
\hline Similarity coefficient & $(X, Y)$ & $(X, Z)$ & $(Y, Z)$ \\
\hline Proposed & 0.051 & 0.947 & 0.002 \\
Cosine & 1.000 & 0.504 & 0.504 \\
Canberra & 0.065 & 0.307 & 0.026 \\
\hline
\end{tabular}

similarity value, since these two points have the same shape, i.e., this similarity measure is sensitive to the propositional transitivity. However, according to the given size displacements of three data points we see that $\operatorname{similarity}(X, Z) \geq \operatorname{similarity}(X, Y) \geq \operatorname{similarity}(Y, Z)$. Considering the first attribute values of data points $X$ and $Z$, the Canberra coefficient returns maximum dissimilarity value 1 . However this returned value does not reveal the original similarity according to their size displacement.

\subsection{New dissimilarity functions for mixed data}

It is now straightforward to define mixed-type dissimilarity function by replacing the squared Euclidean distance and simple matching distance functions in Eq. (1) with Eqs. (11) and (6), respectively, and it is defined as follows:

$$
\operatorname{dist}_{f}\left(x_{1}, x_{2}\right)=\operatorname{dist}_{n}\left(x_{1}^{n}, x_{2}^{n}\right)+\operatorname{dist}_{h}\left(x_{1}^{c}, x_{2}^{c}\right)
$$

where the first term is Minkowski-distance-based dissimilarity function (Eq. (11)) adopted for numerical attribute space and the second term is weighted hamming dissimilarity function (Eq. (6)) adopted for categorical attribute space. We can see that the distance function (Eq. (14)) for mixed-type data falls in the interval $[0, m]$ where ' 0 ' indicates maximum similarity and ' $m$ ' indicates minimum similarity. It may be noted that this distance function for mixed data has equal weightage for either-type attributes since it is defined on the same scale with respect to their dimensionality. To speed up the clustering process, we use the following distance function in initial allocation step of the $k$-prototypes algorithm: 


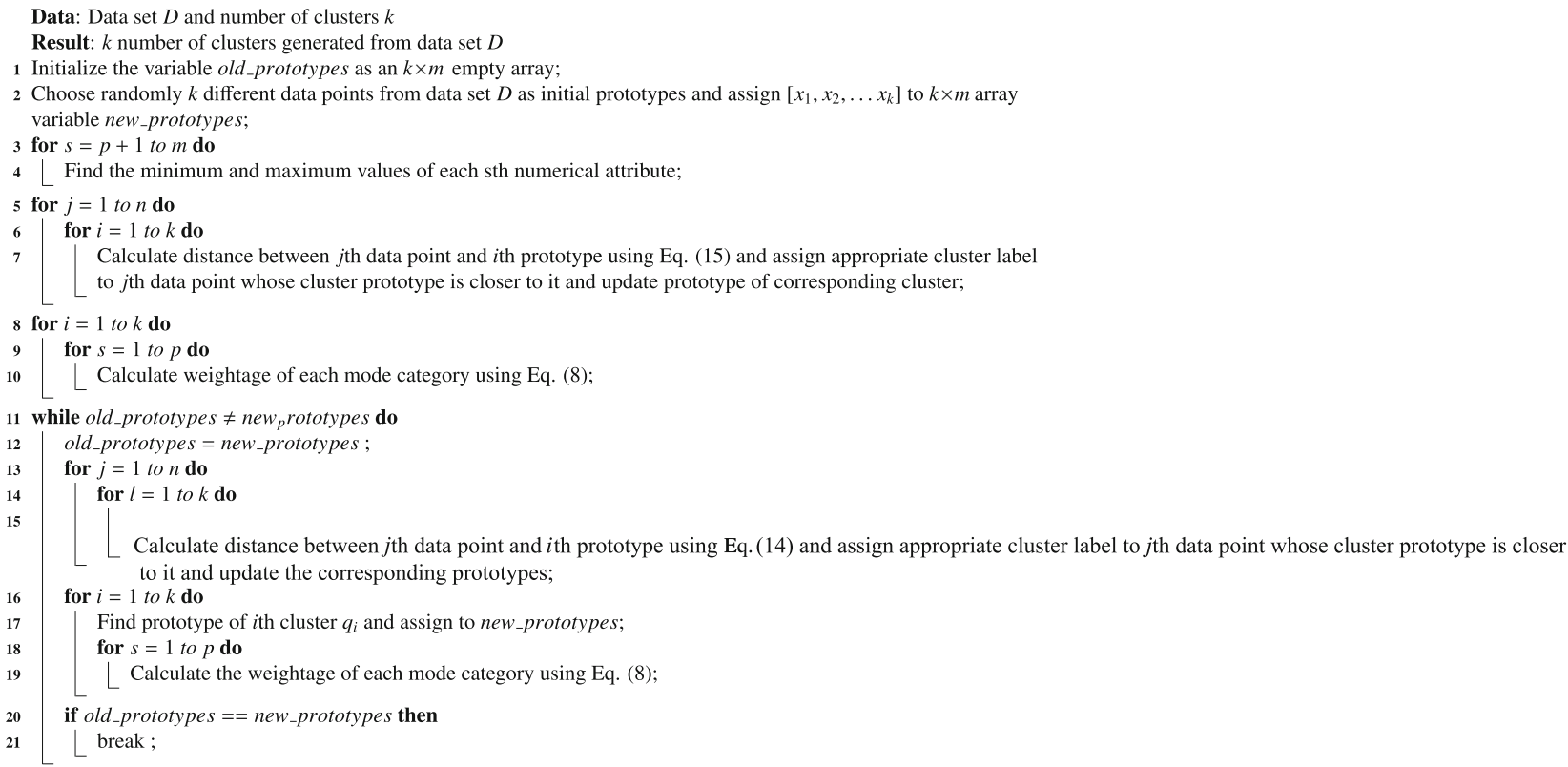

Algorithm 1: $k$-prototype algorithm with the proposed dissimilarity measure.

$$
\operatorname{dist}_{i}\left(x_{1}, x_{2}\right)=\operatorname{dist}_{n}\left(x_{1}^{n}, x_{2}^{n}\right)+\sum_{s=1}^{p} d_{c}\left(A_{s, 1}^{c}, A_{s, 2}^{c}\right)
$$

where the first term is Minkowski-based dissimilarity function (Eq. (11)) adopted for numerical attribute space and the second term is standard simple matching distance function (Eq. (2)) adopted for categorical attribute space. The distance function given in Eq. (15) is also defined on the same scale for either-type attributes with respect to their dimensionality. The cost function given in Eq. (3) can be redefined using the distance function (Eq. (14)) and it is given as follows:

$$
C(W, Q)=\sum_{i=1}^{k} \sum_{j=1}^{n} w_{j i} \operatorname{dist}_{f}\left(x_{j}, q_{i}\right) .
$$

The two dissimilarity functions in this cost function are non-negative and the sequence $C(\bullet, \bullet)$ generated by the $k$ prototypes is strictly decreasing. After a finite number of iterations the algorithm converges to a local minimum point [18].

\subsection{Time complexity analysis}

In this section, we analyse the time complexity of the $k$ prototypes algorithm with new dissimilarity measure while referring to the pseudo-code as shown in Algorithm 1. With respect to each mode category, the time complexity for computing the weightage of mode category according to Eq. (8) is $\mathrm{O}(n)$ and for all mode categories in all clusters, the time complexity is $\mathrm{O}(p n k)$ (see lines 8-10 and 16-19 in Algorithm 1). The computational complexity for assigning all the data points to appropriate clusters in the cluster initialization step is $\mathrm{O}(n m k)$ (see lines 3-7 in Algorithm 1). The time complexity to update the clusters prototypes is $\mathrm{O}(\mathrm{nmk})$ (see lines 16-19 in Algorithm 1). Thus the total time complexity of initial allocation of data points is $\mathrm{O}(n m k)+\mathrm{O}(n p k)=\mathrm{O}(n m k)$ (see lines 3-10 in Algorithm 1).

Suppose that the total number of iterations carried out to break the main loop (see lines 11-21 in Algorithm 1) is $I$; then the total time complexity of the algorithm with new dissimilarity metric is $\mathrm{O}(n m k)+\mathrm{I}(\mathrm{O}(n m k)+\mathrm{O}(n m k)+$ $\mathrm{O}(p n k))=\mathrm{O}(\operatorname{Inmk})$. We see that the time complexity of $k$ prototypes with new dissimilarity measure is linearly scalable with respect to the number of data points, number of clusters and the number of attributes.

\section{Experimental results}

In this section, we evaluate the accuracy and scalability of our proposed scheme using simulations. In section 5.1, we provide the details about simulation environment. In section 5.2, we evaluate the scalability of our scheme on synthetic data sets. In section 5.3, we evaluate the accuracy of our proposed scheme on the real world data sets.

\subsection{Simulation environment}

We have implemented our algorithm using $\mathrm{C}++$ language and executed on an Intel i5 processor $(2.5 \mathrm{GHz})$ with 4 GB memory running on windows 7 operating system. We have compared the scalability and accuracy of our method with that of the original $k$-prototypes 
algorithm [18] and the improved $k$-prototypes (IK-prototypes) algorithm [32].

\subsection{Scalability evaluation of k-prototypes with new dissimilarity coefficient}

We evaluate the scalability of our method with respect to number of records, dimensionality and number of clusters on synthetic data sets. All the synthetic data sets used in our experiments have been generated using a data set generator [37]. All the generated numerical attribute values are in the range of $0-25$ and each categorical attribute domain possesses 8 different categorical values.

5.2a Scalability evaluation with respect to data set size: In this scalability test, we have fixed the data set dimensionality to 20 with equal number of either-type attributes and the number of clusters as 3 , i.e., $k=3$. The data set size varies from 10,000 to 70,000 records. We observe from figure 4 that all the three techniques are almost linear with respect to data set size. Our technique has comparatively similar execution time as those of the original $k$-prototypes. With the new dissimilarity measure, it has better execution time than those of the IK-prototypes.

$5.2 \mathrm{~b}$ Scalability evaluation with respect to dimensionality: In this scalability test, we have fixed the data set size to 30,000 records and the number of clusters as 3, i.e., $k=3$. The dimensionality of data set varies from 10 to 70 . In all cases, we have taken equal number of either-type attributes. We see from figure 5 that on increasing the number of dimensions our scheme behaves similar to the $k$-prototypes and it is better than the IK-prototypes algorithm.

5.2c Scalability evaluation with respect to number of clusters: In this scalability test, we have fixed the data set size to 30,000 records and dimensionality as 30 with equal number of either-type attributes. The number of clusters varies from 3 to 12 . The results are shown in figure 6 . On increasing the number of clusters, our algorithm behaves very much similar to the $k$-prototypes, which is almost constant and much better than the IK-prototypes. Based on these results, we can say that both $k$-prototypes with old and proposed dissimilarity measures consume almost the same amount of time, but with the new dissimilarity measure it results in good accuracy against the original $k$-prototypes method.

\subsection{Accuracy evaluation of proposed scheme on real data sets}

In this evaluation test, we have conducted the experiments on six real data sets taken from the UCI repository [38]. The details of six real data sets are described in table 3 . The accuracy of the $k$-prototypes with the proposed dissimilarity has been compared to those of the original $k$ prototypes and IK-prototypes algorithms. We have computed the accuracy of clustering results using the following:

$$
A C=\frac{\sum_{i=1}^{n} a_{i}}{n}
$$

where $a_{i}$ is number of data points correctly classified with respect to the given class of data points in $i$ th cluster and ' $n$ ' is total number of records.

The results on real world mixed and categorical data sets have been summarized in tables 4 and 5, respectively. It is to be mentioned that the accuracy value in each row of tables has been obtained over the average of 20 runs of algorithm. While performing the $k$-prototypes and

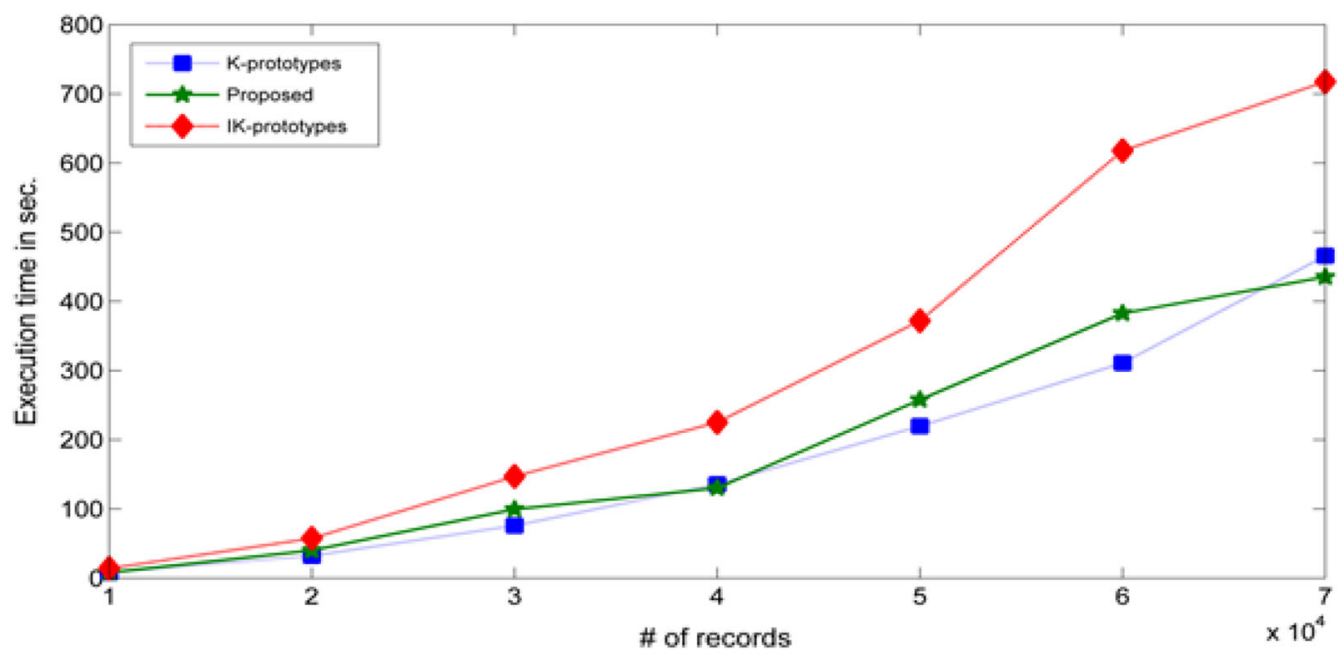

Figure 4. Scalability of proposed method with respect to data set size. 


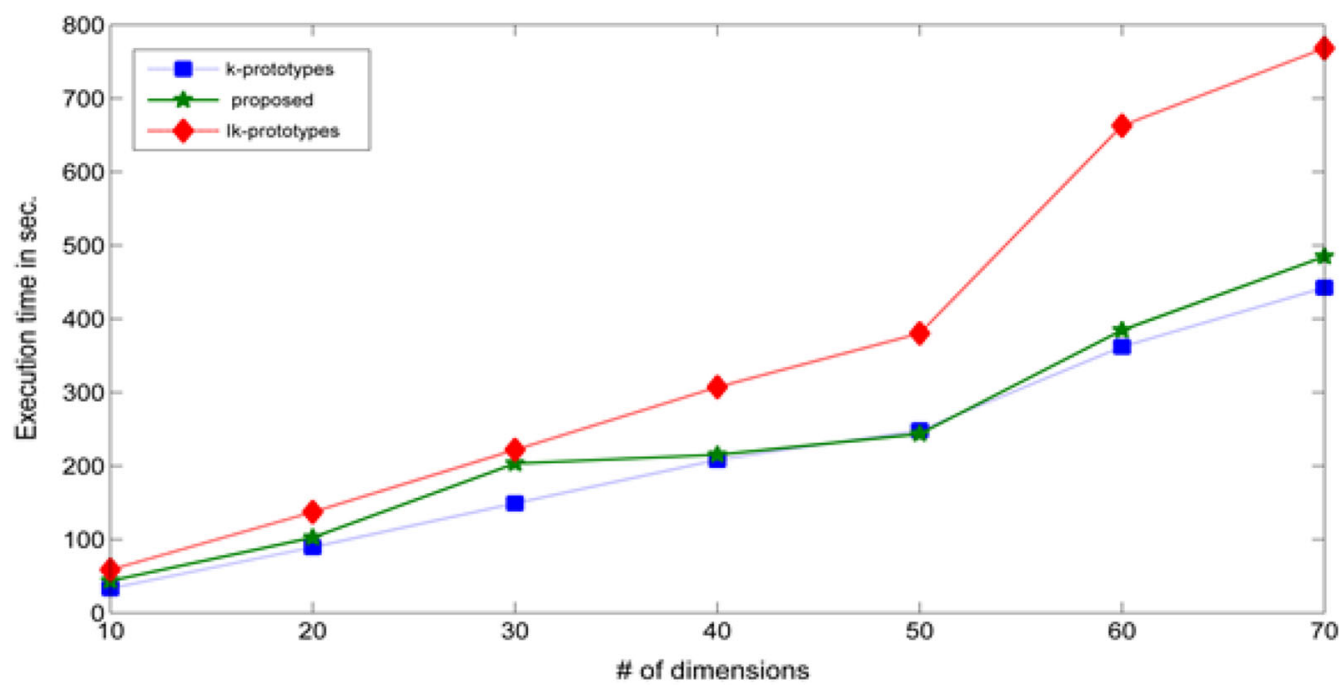

Figure 5. Scalability of proposed method with respect to data set dimensionality.

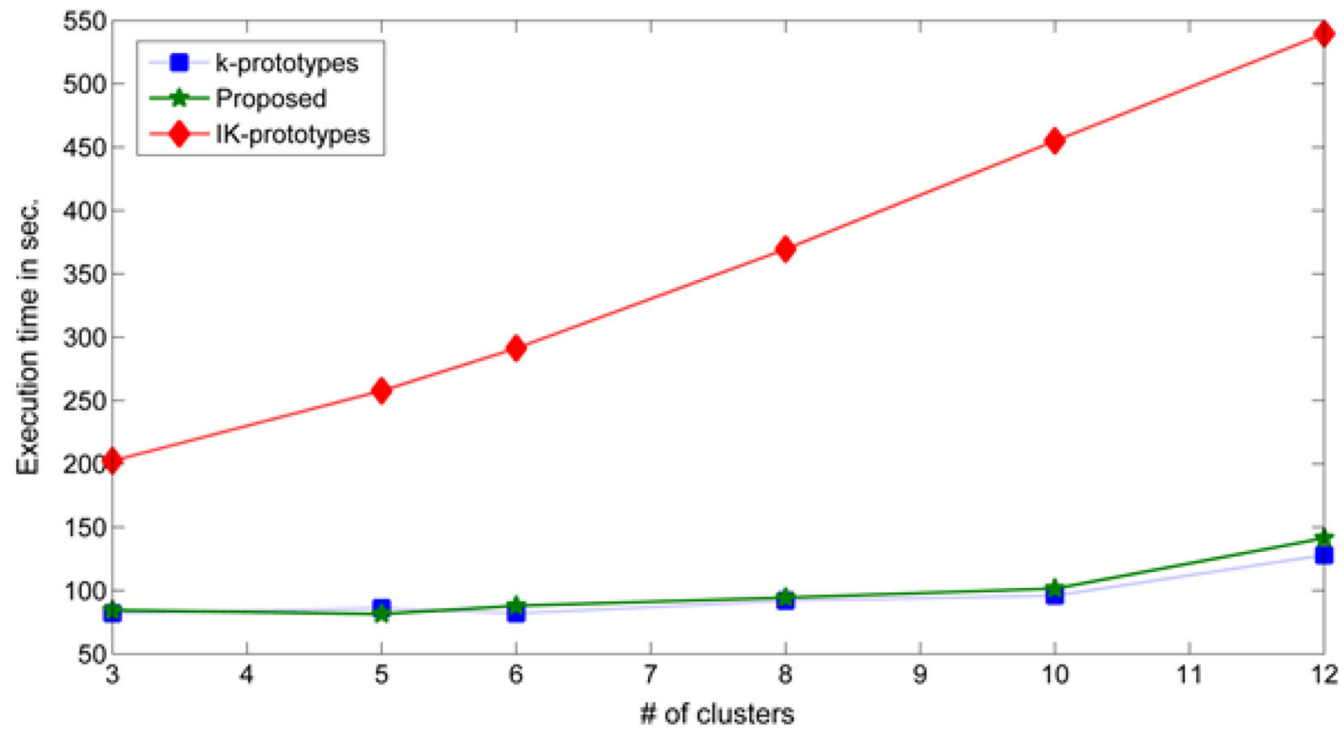

Figure 6. Scalability of proposed method with respect to \# of clusters.

Table 3. Summary details of real data sets.

\begin{tabular}{lccccc}
\hline & \multicolumn{2}{c}{$\#$ of attributes } & & \\
\cline { 2 - 4 } Data set & $\begin{array}{c}\text { Categorical } \\
(\mathrm{p})\end{array}$ & $\begin{array}{c}\text { Numerical } \\
(m-p)\end{array}$ & $\begin{array}{c}\text { \# of } \\
\text { records }\end{array}$ & $\begin{array}{c}\text { \# of missing } \\
\text { values }\end{array}$ & $\begin{array}{c}\text { Average standard deviation of numerical } \\
\text { attributes }(\sigma)\end{array}$ \\
\hline Adult data & 8 & 6 & 32561 & 4262 & 22672.84 \\
Credit approval & 9 & 6 & 690 & 67 & 901.50 \\
Contraceptive method & 7 & 2 & 1473 & 0 & 5 \\
$\quad$ choice & 8 & 0 & 12960 & 0 & - \\
Nursery & 22 & 0 & 8124 & 2480 & - \\
Mushroom & 6 & 0 & 1728 & 0 & - \\
Car evaluation & & & & \\
\hline
\end{tabular}


Table 4. Summary of cluster accuracy on three real world mixed data sets.

\begin{tabular}{lccccccccc}
\hline & \multicolumn{3}{c}{$k$-prototypes } & & \multicolumn{3}{c}{ IK-prototypes } & Proposed \\
\cline { 2 - 3 } Data set & $\gamma=0.7 \sigma$ & $\gamma=0.8 \sigma$ & $\gamma=0.9 \sigma$ & & $\gamma=0.7 \sigma$ & $\gamma=0.8 \sigma$ & $\gamma=0.7 \sigma$ & N/A \\
\hline Adult data & 0.823 & 0.789 & 0.824 & & 0.799 & 0.797 & 0.828 & 0.848 \\
Credit approval & 0.667 & 0.703 & 0.625 & & 0.713 & 0.739 & 0.703 & 0.749 \\
Contraceptive method choice & 0.563 & 0.556 & 0.559 & & 0.646 & 0.649 & 0.643 & 0.658 \\
\hline
\end{tabular}

Table 5. Summary of cluster accuracy on three real world categorical data sets.

\begin{tabular}{lccc}
\hline Data set & $k$-prototypes & IK-prototypes & Proposed \\
\hline Nursery & 0.682 & 0.701 & 0.713 \\
Mushroom & 0.785 & 0.823 & 0.837 \\
Car evaluation & 0.743 & 0.842 & 0.861 \\
\hline
\end{tabular}

IK-prototypes clustering algorithms on adult, credit approval and contraceptive method choice data sets, we have set the weightage parameter $\gamma$ to three different values, i.e., $0.7 \sigma$, $0.8 \sigma$ and $0.9 \sigma$, where $\sigma$ is standard deviation of numerical attributes. There are a number of missing attribute values in data sets that have been replaced with the most frequent attribute values. It may be noted that in the experiments on mushroom, nursery, and car evaluation data sets, we have fixed $\gamma$ value as 1 and there is no change in its value since the data sets are described only by the categorical attributes.

From tables 4 and 5, we see that our proposed method outputs better stable results in terms of clustering accuracy than the $k$-prototypes and IK-prototypes clustering algorithms. Based on all empirical results, we conclude that our proposed dissimilarity coefficient for $k$-prototypes algorithm gives more stable clustering accuracy results while retaining its linear scalability.

\section{Conclusion}

The $k$-prototypes is a widely used clustering algorithm in mixed data domain. The clustering accuracy of any clustering algorithm depends on the similarity or dissimilarity measure used in that algorithm. In this paper, the limitations of dissimilarity measure used in the $k$-prototypes algorithm have been discussed with some illustrative examples. Based on the Minkowski distance metric, a new dissimilarity measure has been adopted for numerical attributes. In addition, the weightage hamming distance measure has been adopted for categorical attribute set based on the relative frequency and distribution of mode categories. The (dis)similarity coefficient for either type of attributes has been defined with the same scale (i.e., equally biased either-type attributes), which is beneficial to clustering analysis on the mixed-type data. Our dissimilarity coefficient preserves the characteristics of clustering, i.e., both high intra-cluster similarity and low inter-cluster similarity. The experimental results on synthetic and real world data sets have shown the efficacy of our scheme. In future work, we intend to extend this algorithm to cluster mixed-type data in stream environment using sliding-window technique.

\section{References}

[1] Chen M S, Han J and Yu P S 1996 Data mining: an overview from a database perspective. IEEE Transactions on Knowledge and Data Engineering 8(6): 866-883

[2] Jain A K, Duin R P W and Mao J 2000 Statistical pattern recognition: a review. IEEE Transactions on Pattern Analysis and Machine Intelligence 22(1): 4-37

[3] Masulli F and Schenone A 1999 A fuzzy clustering based segmentation system as support to diagnosis in medical imaging. Artificial Intelligence in Medicine 16(2): 129-147

[4] Chen L, Zou L J, and Tu L 2012 A clustering algorithm for multiple data streams based on spectral component similarity. Information Sciences 183(1): 35-47

[5] Krishna K, Ramakrishnan K R and Thathachar M A L 1997 Vector quantization using genetic k-means algorithm for image compression. In: IEEE Proceedings of International Conference on Information Communications and Signal Processing, vol. 3, pp. 1585-1587

[6] Charikar M, Chekuri C, Feder T and Motwani R 2004 Incremental clustering and dynamic information retrieval. SIAM Journal on Computing 33(6): 1417-1440

[7] Han J, Pei J and Kamber M 2011 Data mining: concepts and techniques. Elsevier

[8] Anderberg M R 2014 Cluster analysis for applications: probability and mathematical statistics: a series of monographs and textbooks. Academic Press

[9] MacQueen J 1967 Some methods for classification and analysis of multivariate observations. In: Proceedings of the Fifth Berkeley Symposium on Mathematical Statistics and Probability, vol. 1(14), pp. 281-297

[10] Dunn J C 1973 A fuzzy relative of the ISODATA process and its use in detecting compact well-separated clusters. Journal of Cybernetics 3: 32-57

[11] Huang Z 1997 A fast clustering algorithm to cluster very large categorical data sets in data mining. Data Mining and Knowledge Discovery 3(8): 34-39 
[12] Huang Z and Ng M K 1999 A fuzzy k-modes algorithm for clustering categorical data. IEEE Transactions on Fuzzy Systems 7(4): 446-452

[13] Guha S, Rastogi R and Shim K 1999 ROCK: a robust clustering algorithm for categorical attributes. In: IEEE Proceedings of the Fifteenth International Conference on Data Engineering, pp. 512-521

[14] Barbara D, Li Y and Couto J 2002 COOLCAT: an entropybased algorithm for categorical clustering. In: ACM Proceedings of the Eleventh International Conference on Information and Knowledge Management, pp. 582-589

[15] Hsu C C and Chen Y C 2007 Mining of mixed data with application to catalog marketing. Expert Systems with Applications 32(1): 12-23

[16] Li C and Biswas G 2002 Unsupervised learning with mixed numeric and nominal data. IEEE Transactions on Knowledge and Data Engineering 14(4): 673-690

[17] Huang Z 1997 Clustering large data sets with mixed numeric and categorical values. In: Proceedings of the 1st PacificAsia Conference on Knowledge Discovery and Data Mining (PAKDD), pp. 21-34.

[18] Huang Z 1998 Extensions to the k-means algorithm for clustering large data sets with categorical values. Data Mining and Knowledge Discovery 2(3): 283-304

[19] Berkhin P 2006 A survey of clustering data mining techniques. In: Grouping multidimensional data, pp. 25-71

[20] Gan G, Ma C and Wu J 2007 Data clustering: theory, algorithms, and applications. Society for Industrial and Applied Mathematics

[21] Jain A K, Murty M N and Flynn P J 1999 Data clustering: a review. ACM Computing Surveys (CSUR) 31(3): 264-323

[22] Xu R and Wunsch D 2005 Survey of clustering algorithms. IEEE Transactions on Neural Networks 16(3): 645-678

[23] Goodall D W 1966 A new similarity index based on probability. Biometrics 22(4): 882-907

[24] $\mathrm{He} \mathrm{Z,} \mathrm{Xu} \mathrm{X} \mathrm{and} \mathrm{Deng} \mathrm{S} 2005$ Scalable algorithms for clustering large datasets with mixed type attributes. International Journal of Intelligent Systems 20(10): 1077-1089

[25] He Z, Xu X and Deng S 2002 Squeezer: an efficient algorithm for clustering categorical data. Journal of Computer Science and Technology 17(5): 611-624

[26] David G and Averbuch A 2012 SpectralCAT: categorical spectral clustering of numerical and nominal data. Pattern Recognition 45(1): 416-433
[27] Luo H, Kong F and Li Y 2006 Clustering mixed data based on evidence accumulation. In: Advanced data mining and applications. Berlin-Heidelberg: Springer, pp. 348-355

[28] Cheeseman P and Stutz J 1996 Bayesian classification (AutoClass): theory and results. In: Advances in knowledge discovery and data mining, pp. 61-83

[29] Chiu T, Fang D, Chen J, Wang Y and Jeris C 2001 A robust and scalable clustering algorithm for mixed type attributes in large database environment. In: Proceedings of the Seventh ACM SIGKDD International Conference on Knowledge Discovery and Data Mining, ACM, pp. 263-268

[30] Chen H L, Chuang K T and Chen M S 2008 On data labeling for clustering categorical data. IEEE Transactions on Knowledge and Data Engineering 20(11): 1458-1472

[31] Cheung Y M and Jia H 2013 Categorical-and-numerical-attribute data clustering based on a unified similarity metric without knowing cluster number. Pattern Recognition 46(8): 2228-2238

[32] Ji J, Bai T, Zhou C, Ma C and Wang Z 2013 An improved k-prototypes clustering algorithm for mixed numeric and categorical data. Neurocomputing 120: 590-596

[33] San O M, Huynh V N and Nakamori Y 2004 An alternative extension of the k-means algorithm for clustering categorical data. International Journal of Applied Mathematics and Computer Science 14: 241-247

[34] He Z, Deng S and Xu X 2005 Improving k-modes algorithm considering frequencies of attribute values in mode. In: Computational intelligence and security. Berlin-Heidelberg: Springer, pp. 157-162

[35] Ng M K, Li M J, Huang J Z and He Z 2007 On the impact of dissimilarity measure in k-modes clustering algorithm. IEEE Transactions on Pattern Analysis and Machine Intelligence 29(3): 503-507

[36] Rokach L 2005 A survey of clustering dlgorithms. In: Maimon $\mathrm{O} \mathrm{Z}$ and Rokach L (Eds.) Data mining and knowledge discovery handbook. New York: Springer

[37] Gabor M 1999 The datgen dataset generator. http://www. datasetgenerator.com

[38] Bache K and Lichman M 2013 UCI machine learning repository. http://archive.ics.uci.edu/ml. Irvine, CA: University of California, School of Information and Computer Science 